\title{
Developing a career in health professions education: opportunities and challenges
}

\author{
Rashmi Vyas \\ Assistant Vice President Education, Director FAIMER Institute, Foundation for Advancement of International Medical \\ Education and Research (FAIMER), Philadelphia, USA
}

\section{Corresponding Author:}

Email: rvyas@faimer.org

The last decade has seen a shift in the needs of the learner, roles of the teacher and in the health care delivery which has implications for the education of the health professionals. The health care delivery has been evolving towards evidence based practice and there is increasing ease of availability of information to the students. The students need teachers to be facilitators for learning, guiding them to select the best evidence and apply their knowledge for better patient care. Full time committed health professions educators are needed to make health professions education responsive to the changes.

A career in health professions education is both challenging and rewarding. The prospect of being part of the change is exciting. It is a very satisfying experience to play a pivotal role in improving health professions education. There are opportunities to teach the teachers of health professionals, create a culture of continuous quality improvement at ones' health professional college, and be involved in policy and decision making at the highest level related to health professions education. Health Professions educators have to be vigilant in the choices they make and the decisions they take as it affects the students and has the potential to impact health of the community.

However, getting academic recognition and institutional support could be a challenge. Clinical work, research and education are considered to be the three legs of health professions. Out of these three it is the education leg which has the least preparation, recognition and support. This could be detrimental to taking health professions education as a career. Developing a career needs creating the field of health professions education with a pathway for promotions and career growth. While in some countries there is formal career path in health professions education, in other countries it is still an evolving field.

Characteristics of improved field of health professions education include professional associations, scholarship and application of new knowledge and skills to education. An example is the growth of the field of health professions education in India. There are four Fellowship programs offered by Foundation for Advancement of International Medical Education and Research (FAIMER) Regional Institutes (www.faimer.org) in India that train health professions education faculty in Education Methods, Leadership and Management, Project Management and Evaluation, and Education Research and Scholarship. In addition the Medical Council of India (MCI) has implemented basic course and advance course in medical education for medical faculty through MCI recognized medical schools as regional and nodal centers respectively. Development of critical mass of faculty trained through these programs led to the annual meeting of health professions educators called the National Conference in Health Professions Education (NCHPE) which led to the formation of the Academy of Health Professions Educators (AHPE). Today the NCHPE is hosted under the umbrella of the AHPE.

Aspiring health professions educators may consider using the following suggestions to help them develop careers in the field of health professions education. Identify a mentor from the health professions education who will provide guidance and support in this journey. Take advantage of available opportunities to improve the skills and competencies in health professions education. For example one could be proactive in their continuing professional development by participating in conferences, courses, workshops, fellowship programs, diplomas, masters, $\mathrm{PhD}$ etc. in health professions education. One can create one's own opportunities by innovating, implementing and evaluating one's own ideas. Doing education research and disseminating one's work will bring the academic recognition and contribute significantly to the field. Be part of a community of educators. Be a change agent and lead the change in making health professions education responsive to the needs of the institution and the society. Last but not the least is to have the passion for health professions education. It could start with a dream which is turned into a vision that gets translated into action with passion.

It is crucial that health professions educators are valued. Improving, renewing, elevating health professions education to meet the changes in the needs of the learner, role of the teacher and the evolving health care delivery requires considerable time, effort, training and expertise. Institutions must support health professions educators as they lay the foundation for improving health by improving education. 